\title{
Density of states techniques for lattice field theories using the functional fit approach (FFA)
}

\author{
Christof Gattringer $^{a}$, Mario Giuliani $^{*} a$, Alexander Lehmann ${ }^{a, b}$, Pascal Törek $^{\star} a \dagger$ \\ ${ }^{a}$ Universität Graz, Institut für Physik, Universitätsplatz, 5, 8010 Graz, Austria \\ ${ }^{b}$ Humboldt-Universität zu Berlin, Institut für Physik, 12489 Berlin, Germany \\ E-mail: christof.gattringer@uni-graz.at, mario.giuliani@uni-graz.at, \\ alehmannephysik.hu-berlin.de, pascal.toerek@uni-graz.at,
}

\begin{abstract}
We discuss a variant of density of states (DoS) techniques for lattice field theories, the so-called "functional fit approach" (FFA). The DoS FFA is based on a density of states $\rho(x)$ which is parameterized on small intervals of the argument $x$ of $\rho(x)$. On these intervals restricted Monte Carlo simulations with an additional Boltzmann factor $\exp (\lambda x)$ allow to determine $\rho(x)$ very precisely by obtaining its parameters from fitting the Monte Carlo data to a known function of $\lambda$.

We describe the method in detail and show its applicability in four different systems, three of which have a complex action problem: The SU(3) spin model with a chemical potential, U(1) lattice gauge theory, the $\mathbb{Z}_{3}$ spin model with chemical potential, and 2-dimensional U(1) lattice gauge theory with a topological term. In all cases we compare to reference calculations, which partly were done in a dual formulation where the complex action problem is absent. In all four cases we find a very encouraging performance of the DoS FFA.
\end{abstract}

The 33rd International Symposium on Lattice Field Theory

14 -18 July 2015

Kobe International Conference Center, Kobe, Japan

\footnotetext{
${ }^{*}$ Speaker.

$\dagger$ This work is based on the presentations by Mario Giuliani and Pascal Törek in the parallel sessions. It is supported by the Austrian Science Fund FWF, through the DK Hadrons in Vacuum, Nuclei, and Stars (FWF DK W1203-N16) and by Grant I 1452-N27. Partial support from DFG TR55, "Hadron Properties from Lattice QCD" is acknowledged. We thank Ydalia Delgado Mercado, Kurt Langfeld, Biagio Lucini, Axel Maas, Michael Müller-Preussker and Uwe-Jens Wiese for discussions and comments on the literature.
} 


\section{Introduction}

It is well known that Monte Carlo simulations of lattice field theories at finite density or with a topological term are plagued by the complex action problem: The action $S$ has an imaginary part and the Boltzmann factor $e^{-S}$ cannot be used as a probability weight in a stochastic process. A possible way out is the density of states approach, where a density $\rho(x)$ is computed in a simulation without the imaginary part of the action which is then included in a subsequent reweighting step. Different variants of density of states techniques have seen quite some attention recently [1]-[5].

The main technical challenge of the density of states (DoS) approach is the determination of $\rho(x)$ with very high accuracy, which is necessary because in the reweighting step for including the complex phase the density is probed with a highly oscillating factor. Achieving the required high accuracy is further complicated by the fact that $\rho(x)$ varies over many orders of magnitude and in some parameter ranges also the tails of $\rho(x)$ give a sizable contribution to physical observables. Consequently the recent developments have focussed on achieving maximal accuracy for $\rho(x)$.

In this contribution we present and test an approach which we refer to as "functional fit approach" (FFA). The argument $x$ of the density is divided into small intervals and the density $\rho(x)$ is parameterized on each of these intervals. Subsequently one performs a restricted Monte Carlo simulation on each of the intervals and probes the system with an additional Boltzmann factor $e^{\lambda x}$, where the free parameter $\lambda$ is used to probe the system. The parameters of $\rho(x)$ on the given interval can be determined from fitting the restricted Monte Carlo data on the interval with a known function of $\lambda$.

\section{General scheme of the functional fit approach}

\subsection{Definition of the density}

The basic equations for computing an observable $\langle O\rangle$ and the partition function $Z$ in a lattice field theory are given by

$$
\langle O\rangle=\frac{1}{Z} \int D[\psi] e^{-S[\psi]} O[\psi] \quad, \quad Z=\int D[\psi] e^{-S[\psi]} .
$$

Here we use $\psi$ as a generic symbol for all fields of the theory (which for simplicity we assume to be bosonic here), and $S[\psi]$ is the corresponding Euclidean lattice action. The observable is a functional $O[\psi]$ of the field configurations $\psi$. By $\int D[\psi]$ we denote the lattice path integral, i.e., the product over the measures of the fields at each site (or link) of the finite lattice.

To keep the approach as general as possible we split the action into two parts as follows,

$$
S[\psi]=S_{\rho}[\psi]+\xi X[\psi]
$$

Both parts $S_{\rho}[\psi]$ and $X[\psi]$ are real functionals of the fields $\psi$ and $\xi$ is a parameter which is either real or purely imaginary, where the latter case is the one relevant for a situation with a complex action problem. Here we use a weighted density of states and $S_{\rho}[\psi]$ is that part of the action which we include in the density. The second part $X[\psi]$ is then taken into account via reweighting, and in case of an imaginary $\xi$, this is how the complex action problem is handled. We remark that 
even more general decompositions of the action are possible, where only part of the real part of the action is used in the weighted density and the rest of the real part is included via reweighting.

Two examples for a decomposition according to (2.2) are:

- Charged scalar field $\phi$ with a chemical potential $\mu$ :

$$
S_{\rho}[\phi]=\operatorname{Re} S[\phi] \quad, \quad \xi X[\phi]=i \operatorname{Im} S[\phi] \quad, \quad \xi=i \sinh (\mu),
$$

where $S_{\rho}$ and $X$ are essentially the real and imaginary parts of the action and the control parameter $\xi$ is given by $\xi=i \sinh (\mu)$.

- Yang-Mills theory with a topological term:

$$
S_{\rho}[U]=S_{G}[U] \quad, \quad X[U]=Q[U] \quad, \quad \xi=i \theta .
$$

We use $U$ to denote the configurations of the lattice gauge fields and $S_{G}[U]$ is the gauge action. $Q[U]$ is a discretization of the topological charge and $\theta$ the vacuum angle.

The next step is to introduce a weighted density of states, defined as

$$
\rho(x)=\int D[\psi] e^{-S_{\rho}[\psi]} \delta(X[\psi]-x) .
$$

Obviously we obtain for the partition sum $Z$ and for observables $O$ which are functions $O[X]$ :

$$
Z=\int_{x_{\min }}^{x_{\max }} d x \rho(x) e^{-\xi x}, \quad\langle O\rangle=\frac{1}{Z} \int_{x_{\min }}^{x_{\max }} d x \rho(x) e^{-\xi x} O[x]
$$

where $x_{\min }$ and $x_{\max }$ denote the bounds of $X[\psi]$. Usually there is a symmetry transformation of the fields, $\psi \rightarrow \psi^{\prime}$, with $S_{\rho}\left[\psi^{\prime}\right]=S_{\rho}[\psi], X\left[\psi^{\prime}\right]=-X[\psi]$ and $\int D\left[\psi^{\prime}\right]=\int D[\psi]$. Such a symmetry guarantees the reality of $Z$ and implies that $\rho(x)$ is an even function,

$$
\rho(-x)=\int D[\psi] e^{-S_{\rho}[\psi]} \delta(X[\psi]+x)=\int D\left[\psi^{\prime}\right] e^{-S_{\rho}\left[\psi^{\prime}\right]} \delta\left(-X\left[\psi^{\prime}\right]+x\right)=\rho(x) .
$$

It is easy to show that the expressions for $Z$ and $\langle O\rangle$ simplify to

$$
Z=2 \int_{0}^{x_{\max }} d x \rho(x) \cosh (\xi x),\langle O\rangle=\frac{2}{Z} \int_{0}^{x_{\max }} d x \rho(x)\left(\cosh (\xi x) O_{e}[x]-\sinh (\xi x) O_{o}[x]\right),
$$

where we have defined the even and odd parts of the observable as $O_{e}[x]=(O[x]+O[-x]) / 2$, $O_{o}[x]=(O[x]-O[-x]) / 2$. Note that for purely imaginary $\xi$ the sinh and cosh are replaced by sin and cos. Eq. (2.8) shows that we need $\rho(x)$ only for positive $x \in\left[0, x_{\max }\right]$.

\subsection{Parametrization of the density}

The next step is to find a suitable parametrization of the density $\rho(x)$, which in many cases is an exponential ansatz which we discuss now. For the parametrization we first divide [6] the range $\left[0, x_{\max }\right]$ into $N$ intervals of variable size $\Delta_{i}, i=0,1 \ldots N-1$ with $\sum_{i=0}^{N-1} \Delta_{i}=x_{\max }$. For the boundaries 
of the intervals we introduce the notation $x_{n}=\sum_{i=0}^{n-1} \Delta_{i}$, such that the $n$-th interval is $\left[x_{n}, x_{n+1}\right]$. The exponential ansatz for $\rho(x)$ now has the form

$$
\rho(x)=e^{-l(x)} \quad \text { with } \quad l(x)=d_{n}+x k_{n} \quad \text { for } \quad x \in\left[x_{n}, x_{n+1}\right] .
$$

In other words the density is the exponential of a piecewise linear function $l(x)$. For each interval this linear function is parameterized by a constant $d_{n}$ and a slope $k_{n}$. We furthermore require $l(x)$ to be continuous and normalize it to $l(0)=0$, which corresponds to the normalization $\rho(0)=1$ (such a normalization can be chosen freely). The continuity condition and the normalization completely determine the constants $d_{n}$, and the slopes $k_{n}, n=0,1 \ldots N-1$ are the only remaining parameters of $\rho(x)$. A simple exercise gives the explicit representation

$$
l(x)=\sum_{i=0}^{n-1} \Delta_{i}\left(k_{i}-k_{n}\right)+x k_{n} \quad \text { for } \quad x \in\left[x_{n}, x_{n+1}\right] .
$$

We remark that one can use variable interval sizes $\Delta_{i}$ to achieve a finer parametrization of $\rho(x)$ in regions of $x$ where $\rho(x)$ shows a large variation.

\subsection{Determination of the parameters with restricted expectation values}

For the determination of the parameters $k_{n}$ we define so-called restricted expectation values $\langle\langle O\rangle\rangle_{n}(\lambda)$. They depend on a real parameter $\lambda$ and are defined as [3]:

$$
\begin{aligned}
\langle\langle O\rangle\rangle_{n}(\lambda) & =\frac{1}{Z_{n}(\lambda)} \int D[\psi] e^{-S_{\rho}[\psi]+\lambda X[\psi]} O[X[\psi]] \Theta_{n}[X[\psi]], \\
Z_{n}(\lambda) & =\int D[\psi] e^{-S_{\rho}[\psi]+\lambda X[\psi]} \Theta_{n}[X[\psi]], \\
\Theta_{n}[x] & =\left\{\begin{array}{ll}
1 & \text { for } x \in\left[x_{n}, x_{n+1}\right] \\
0 & \text { otherwise }
\end{array} .\right.
\end{aligned}
$$

The function $\Theta_{n}[x]$ restricts the values of $x$ to the interval $\left[x_{n}, x_{n+1}\right]$ - thus the name "restricted expectation values". The parameter $\lambda$ enters the definitions of $\langle\langle O\rangle\rangle_{n}(\lambda)$ and $Z_{n}(\lambda)$ via the additional Boltzmann factor $e^{\lambda X[\psi]}$ and allows to probe the system by varying $\lambda$. In the context of the density of states approach this Boltzmann factor can be used to populate different regions of the density $\rho(x)$ in order to determine it accurately for all $x \in\left[x_{n}, x_{n+1}\right]$. The restricted expectation values $\langle\langle O\rangle\rangle_{n}(\lambda)$ can easily be evaluated in a Monte Carlo simulation with an additional rejection step for configurations where $X[\psi] \notin\left[x_{n}, x_{n+1}\right]$.

One can now also work out $\langle\langle O\rangle\rangle_{n}(\lambda)$ and $Z_{n}(\lambda)$ using the density of states. One finds

$$
\langle\langle O\rangle\rangle_{n}(\lambda)=\frac{1}{Z_{n}(\lambda)} \int_{x_{n}}^{x_{n+1}} d x \rho(x) e^{\lambda x} O[x] \quad, \quad Z_{n}(\lambda)=\int_{x_{n}}^{x_{n+1}} d x \rho(x) e^{\lambda x} .
$$

Particularly simple is the expectation value $\langle\langle X\rangle\rangle_{n}(\lambda)$, which we write as $\langle\langle X\rangle\rangle_{n}(\lambda)=\partial Z_{n}(\lambda) / \partial \lambda$. Using the parametrization (2.9), (2.10) of the density $\rho(x)$, we can explicitly evaluate $Z_{n}(\lambda)$ from (2.12) and thus also $\langle\langle X\rangle\rangle_{n}(\lambda)$. A straightforward calculation gives

$$
\frac{1}{\Delta_{n}}\left(\langle\langle X\rangle\rangle_{n}(\lambda)-x_{n}\right)-\frac{1}{2}=F\left(\left(\lambda-k_{n}\right) \Delta_{n}\right)
$$


where we have introduced the function

$$
F(s)=\frac{1}{1-e^{-s}}-\frac{1}{s}-\frac{1}{2} .
$$

$F(s)$ is a real-valued smooth function of a real variable $s$. One finds $F(0)=0, \lim _{s \rightarrow \pm \infty} F(s)= \pm \frac{1}{2}$ and $F^{\prime}(0)=\frac{1}{12}$. The remarkable fact is that Eq. (2.13) can be used to precisely determine the parameter $k_{n}$ : The left hand side of (2.13) can be evaluated with high accuracy in a restricted Monte Carlo simulation for several values of $\lambda$. The result is then described by the right hand side of (2.13) where $k_{n}$ appears as the only undetermined variable, and we compute $k_{n}$ as the result of a one-parameter fit of the data for the left hand side of (2.13) with the function $F\left(\left(\lambda-k_{n}\right) \Delta_{n}\right)$. Thus the name "functional fit approach" (FFA).

The procedure is then repeated for all intervals $\left[x_{n}, x_{n+1}\right]$ and we obtain all $k_{n}, n=0,1 \ldots N-1$. From the $k_{n}$ we obtain the density $\rho(x)$ via (2.10) and (2.9) and can compute the expectation values of observables using (2.8).

This concludes the general description of the FFA approach and we now discuss its actual application for several examples.

\section{The SU(3) spin system with a chemical potential}

We begin the explicit presentation of the FFA approach by discussing its implementation for the SU(3) spin model with a chemical potential $\mu$. The model is an effective theory for the local Polyakov loop $P(x)$. It has a dual representation [7] and the dual simulation results from [8] will be used as reference data for testing the FFA results. The action of the model is given by

$$
S[P]=\tau \sum_{x} \sum_{v=1}^{3}\left[\operatorname{Tr} P(x) \operatorname{Tr} P(x+\hat{v})^{\dagger}+\text { c.c. }\right]+\kappa \sum_{x}\left[e^{\mu} \operatorname{Tr} P(x)+e^{-\mu} \operatorname{Tr} P(x)^{\dagger}\right] .
$$

Here $x$ runs over the sites of a 3-dimensional lattice with periodic boundary conditions. $\tau$ is a parameter that is an increasing function of the temperature of the effective theory and $\kappa$ is a parameter that is a decreasing function of the quark mass. The dynamical degrees of freedom are the Polyakov loop variables $P(x) \in \mathrm{SU}(3)$ attached to the sites $x$ of the lattice. Since they only enter with their trace, we can use the simplified representation $P(x)=\operatorname{diag}\left(e^{i \theta_{1}(x)}, e^{i \theta_{2}(x)}, e^{-i\left(\theta_{1}(x)+\theta_{2}(x)\right)}\right)$ with only two angles $\theta_{1}(x), \theta_{2}(x) \in[-\pi, \pi]$.

The decomposition of the action into $S_{\rho}[P]$ and $X[P]$ according to (2.2) is straightforward,

$S_{\rho}[P]=\operatorname{Re} S[P], X[P]=\sum_{x}\left[\sin \left(\theta_{1}(x)\right)+\sin \left(\theta_{2}(x)\right)-\sin \left(\theta_{1}(x)+\theta_{2}(x)\right)\right], \quad \xi=i 2 \kappa \sinh (\mu)$.

Up to a factor the functional $X[P]$ is the imaginary part of the action. $X[P]$ is bounded, i.e., $X[P] \in\left[-\frac{3 \sqrt{3}}{2} V ; \frac{3 \sqrt{3}}{2} V\right] \equiv\left[-x_{\max }, x_{\max }\right]$, where $V$ is the number of lattice points. $X[P]$ is odd under the transformation $\theta_{i}(x) \rightarrow-\theta_{i}(x)$, while $S_{\rho}[P]$ and the path integral measure $D[P]$ (the product over the reduced Haar measures) are even, such that (2.8) applies and we need the density $\rho(x)$ only for positive $x$. The density of states is parameterized as in (2.9) and (2.10), and restricted vacuum expectation values are defined as given in (2.11). The parameters $k_{n}$ of the density can be determined from fits as outlined in (2.13), (2.14). 


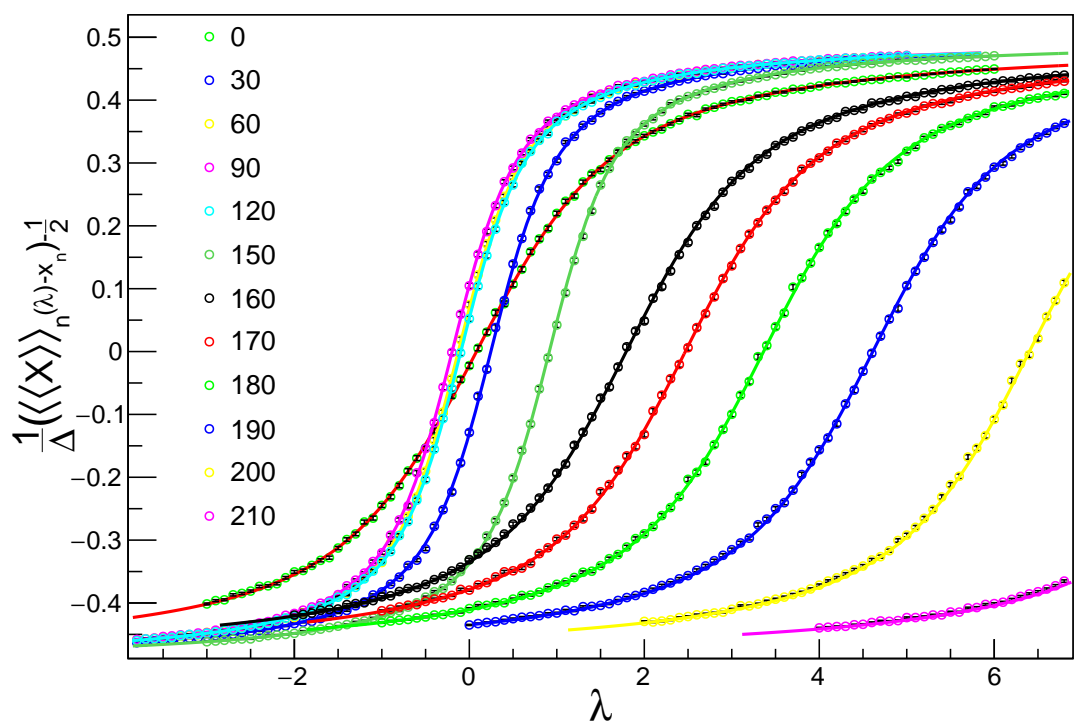

Figure 1: Results for the restricted vacuum expectation values $\left(\langle\langle X\rangle\rangle_{n}(\lambda)-x_{n}\right) / \Delta_{n}-1 / 2$ as a function of $\lambda$ for different intervals $n$ (indicated by the numbers in the legend). The symbols are the Monte Carlo results and the full curves the fits with $F\left(\left(\lambda-k_{n}\right) \Delta_{n}\right)$. We show results for $\tau=0.075, \mu=0.0$. Note that we use two different sizes $\Delta_{n}$ for the intervals, as can be seen from the fact that some of the data sets have a smaller slope near 0 .

For the tests presented here we performed simulations on lattices of size $8^{3}$ at $\kappa=0.005$ and several values of $\tau$ and $\mu$. The statistics used for computing each restricted expectation value in this exploratory study is typically 5000 measurements separated by 10 updates for decorrelation after 2000 equilibration updates. We use between $N=250$ and $N=500$ intervals for dividing $\left[0, x_{\max }\right]$ with typically two different sizes for the $\Delta_{n}$.

We begin the discussion of the numerical results with showing data for the restricted expectation values $\left(\langle\langle X\rangle\rangle_{n}(\lambda)-x_{n}\right) / \Delta_{n}-\frac{1}{2}$, i.e., the lhs. of Eq. (2.14). The corresponding Monte Carlo data, shown as circles in Fig. 1, are for $\tau=0.075, \mu=0.0$, and we give the results for many intervals $n$ as labelled in the legend. The smooth curves are the results for the corresponding fits with $F\left(\left(\lambda-k_{n}\right) \Delta_{n}\right)$. It is obvious that the Monte Carlo data are very well described by these fits. In fact this is an important self-consistency check for the chosen parameterization of the density (compare (2.9)). We remark, that in Fig. 1 two different values were used for the intervals $\Delta_{n}$, which leads to a different slope of the curves near their zeros.

From the fits to the restricted Monte Carlo data as shown in Fig. 1 we obtain all slopes $k_{n}$ and via (2.10) and (2.9) the density $\rho(x)$. Our results for the density are shown in Fig. 2, where we plot $\ln \rho(x)$ as a function of $x$ for $\mu=0.0$ and different values of the temperature parameter $\tau$. The plot shows that the density of states increases considerably when increasing the temperature parameter for states with large $x$, i.e., states with large imaginary parts of the action. For all values of $\tau$ an obvious feature is that the density varies over many orders of magnitude, which illustrates the key challenge of all DoS approaches.

Having determined the density $\rho(x)$ the final step is the evaluation of observables according 


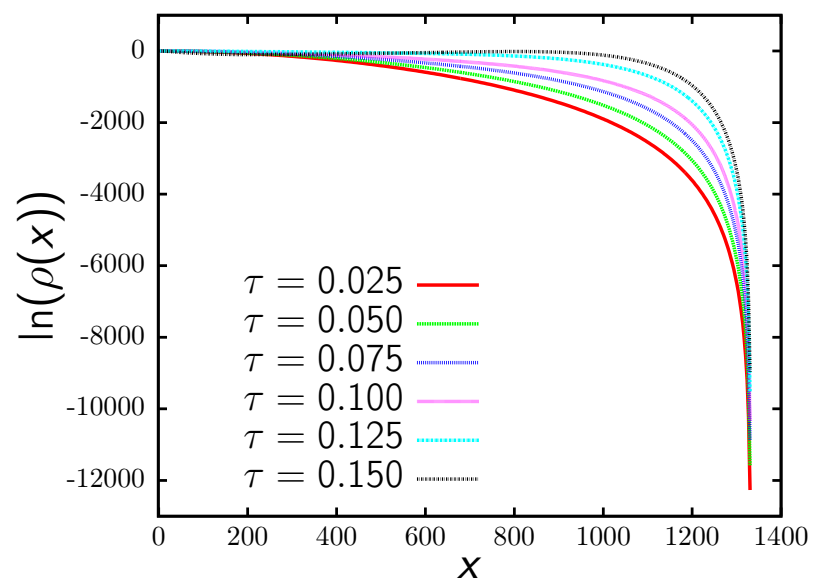

Figure 2: The logarithm of the density $\rho(x)$ versus $x$. We show results for $\mu=0.0$ at several values of $\tau$.

to (2.8). For the SU(3) spin model we consider the particle number density $n$,

$$
n \equiv-\frac{1}{V} \frac{1}{2 \kappa} \frac{\partial}{\partial \sinh \mu} \ln Z=\frac{2}{V} \frac{1}{Z} \int_{0}^{x_{\max }} d x \rho(x) \sin (x 2 \kappa \sinh \mu) x,
$$

and the corresponding susceptibility $\chi_{n}$ obtained by another derivative with respect to $\sinh \mu$. The results for these observables are shown in Fig. 3 as a function of the chemical potential $\mu$. We find very good agreement of the DoS FFA results with the results of the reference calculation with dual variables [8] up to chemical potential values of $\mu \sim 3$ for $n$ and up to $\mu \sim 2.5$ for $\chi_{n}$. We remark that the chemical potential $\mu$ as it appears in the action (3.1) of the SU(3) model is rescaled by the inverse temperature $\beta$ of QCD, which is modeled by the effective $\mathrm{SU}(3)$ theory. In dimensionless units thus the range of good agreement between DoS FFA and dual methods is up to $\mu \beta \sim 3$ for the density and $\mu \beta \sim 2.5$ for $\chi_{n}$, which is rather remarkable for the simple exploratory implementation of FFA discussed here.
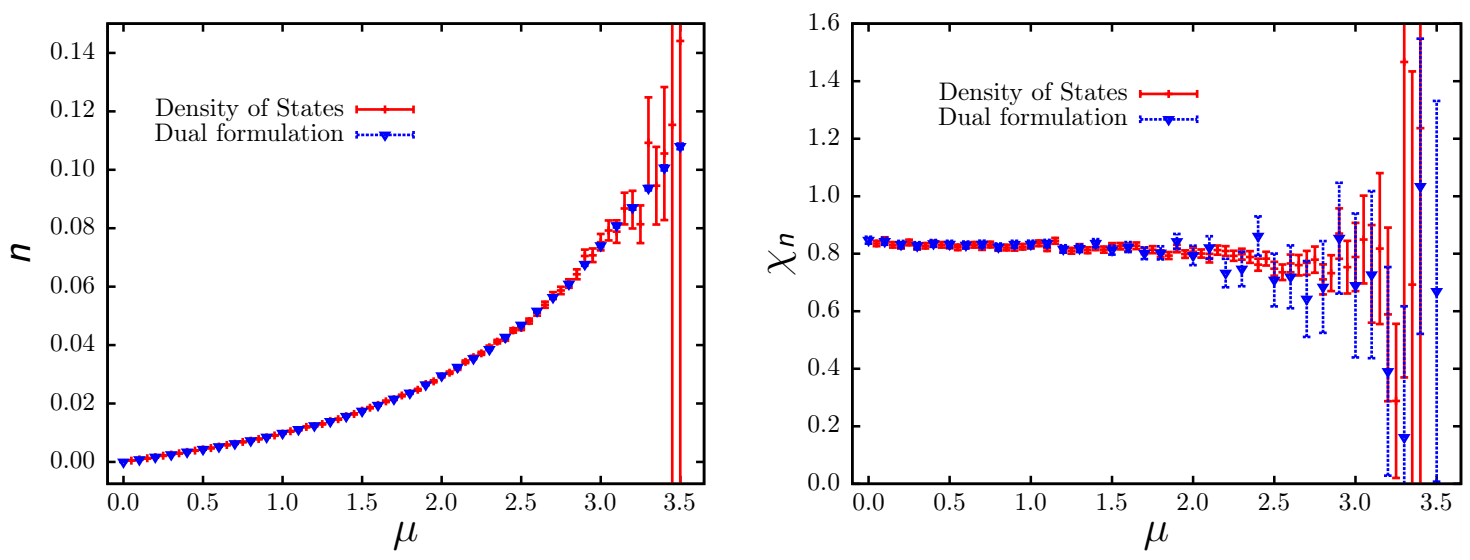

Figure 3: The particle number density $n$ (lhs.) and the particle number susceptibility $\chi_{n}$ (rhs.) versus the chemical potential $\mu$. We show the results for $\tau=0.066, \kappa=0.005$ lattices and compare the data from the FFA (plusses) to the results of a dual simulation (triangles). 


\section{The $\mathbb{Z}_{3}$ spin system with a chemical potential}

We now come to presenting the DoS FFA results for the $\mathbb{Z}_{3}$ spin model in an external field which is closely related to the SU(3) spin system discussed in the previous section. The main difference is that the group $\mathrm{SU}(3)$ of the spins is replaced by its center $\mathbb{Z}_{3}$, such that the dynamical degrees of freedom are the spins $P_{x} \in \mathbb{Z}_{3}=\left\{1, e^{i 2 \pi / 3}, e^{-i 2 \pi / 3}\right\}$, living on the sites $x$ of a 3 -dimensional lattice with periodic boundary conditions. The corresponding action is

$$
S[P]=\sum_{x}\left[\tau \sum_{v=1}^{3}\left(P_{x}^{\star} P_{x+\hat{v}}+\text { c.c. }-2\right)+\kappa e^{\mu \beta}\left(P_{x}-1\right)+\kappa e^{-\mu \beta}\left(P_{x}^{\star}-1\right)\right] .
$$

The action is normalized such that $S[P]=0$ if $P_{x}=1 \forall x$, and here we show explicitly the inverse temperature $\beta$ of the underlying QCD-inspired theory, i.e., the chemical potential is coupled in the dimensionless form $\mu \beta$. The parameters $\tau$ and $\kappa$ play the same role as in the SU(3) model. Similar to the case of the SU(3) model also here one can decompose the action into real and imaginary parts. The Boltzmann factor with the real part is used in the weighted density, while the phase from imaginary part needs to be integrated over the density for computing observables.

There is an important difference to the SU(3) case discussed in the previous section: Here the dynamical degrees, i.e., the $\mathbb{Z}_{3}$ spins, are discrete and the imaginary part which plays the role of the argument $x$ of the density $\rho(x)$ can have only a finite number of discrete values $x=$ $-V,-V+1, \ldots V$ (on a finite volume $V$ ). Thus, parameterizing the density as piecewise constant,

$$
\rho(x)=\prod_{j=0}^{|x|} e^{-a_{j}}=\exp \left(-\sum_{j=0}^{|x|} a_{j}\right), x=-V,-V+1, \ldots . V-1, V,
$$

even allows for an exact representation of the density of states. For determining the parameters $a_{j}$ of the density we use a strategy [5] where we perform overlapping restricted Monte Carlo simulations which also extend to the two neighboring intervals next to the interval $j$ where we want to determine $a_{j}$. Also for this case one can work out the partition sum and the restricted expectation values in terms of the parameterized density, and again the parameters $a_{j}$ can be obtained from fitting the expectation values with a known function of $\lambda$.

Here we present results [5] for $\left\langle M-M^{*}\right\rangle$, where $M=\sum_{x} P_{x}$, as well as for the free energy $F=-\ln Z$. In Fig. 4 we show our results for two different values of the temperature parameter $\tau$, one in the confining phase of the theory, the other one in the deconfined phase (see [9]). In the lhs. column we plot the results for $\tau=0.16$ (confining), while the rhs. column is for $\tau=0.178$ (deconfined). The top row displays $\left\langle M-M^{*}\right\rangle$, the bottom row is for the free energy. We compare the results from plain DoS FFA (circles) to the outcome of the dual simulation from [9] (crosses). For $\left\langle M-M^{*}\right\rangle$ we also show the results after the density was fit with a polynomial (triangles), a strategy which we discuss below.

It is obvious that for the free energy the DoS FFA and the dual results agree perfectly up to $\mu \beta=4.0$. For $\left\langle M-M^{*}\right\rangle$ the agreement is reasonable only for the deconfined data set $(\tau=0.178$, rhs.), while for the confined dataset $(\tau=0.16$, lhs.) the agreement of DoS FFA and dual results breaks down already at $\mu \beta \sim 1$. The reason for this breakdown are small fluctuations in the density coming from the statistical error of the Monte Carlo determination of the density. As $\mu$ is increased, 

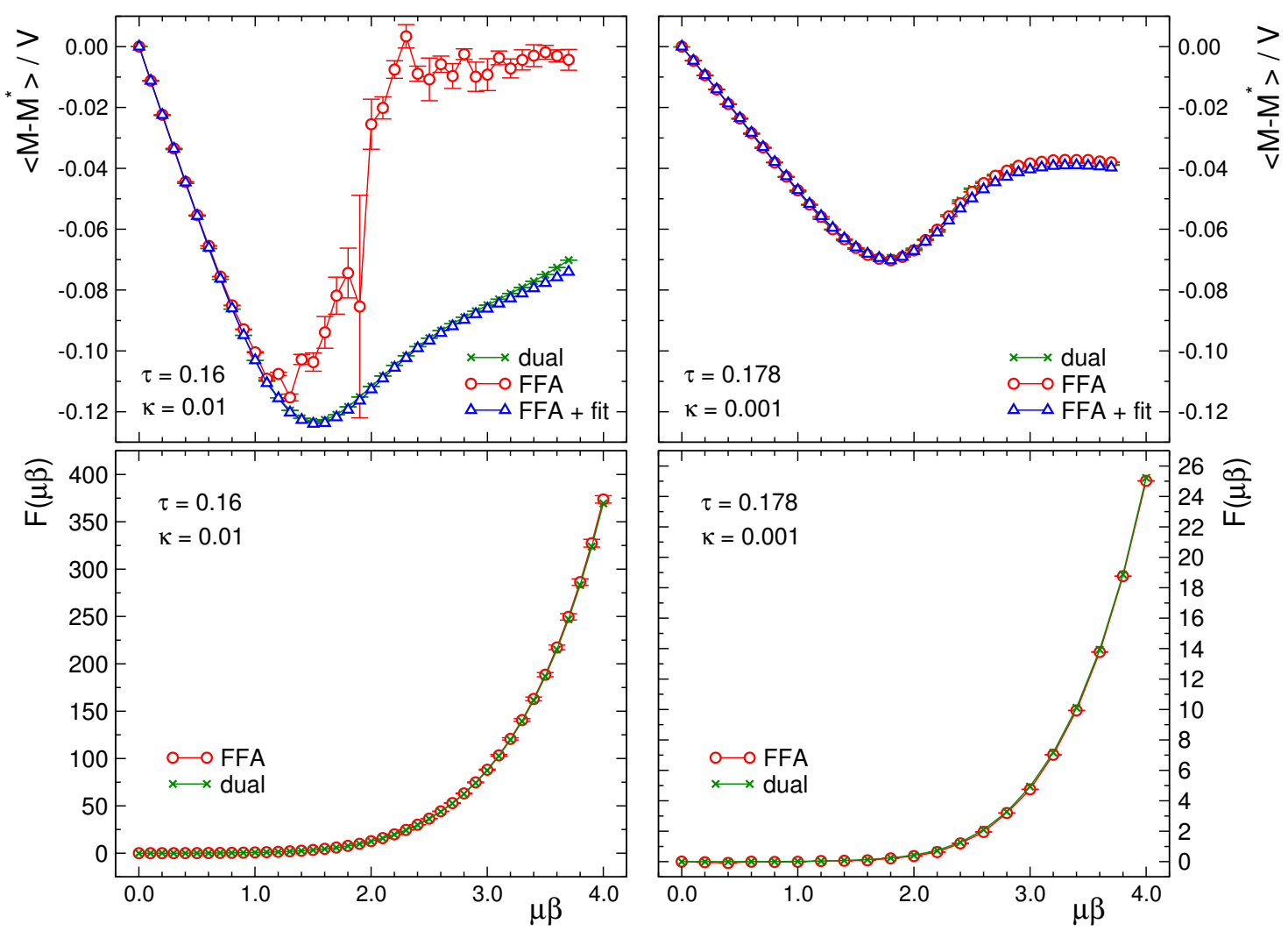

Figure 4: Results for $\left\langle M-M^{*}\right\rangle$ (top row of plots) and the free energy (bottom) as a function of $\mu \beta$. We use two sets of parameters, $\tau=0.16, \kappa=0.01$ on the lhs., and $\tau=0.178$ and $\kappa=0.001$ on the rhs. We show results from the FFA algorithm, the FFA algorithm combined with a fit of $\rho(x)$ (only in the top row of plots) and for comparison also the results from a simulation in the dual representation.

the density is probed by a faster oscillating factor and the fluctuations start to spoil the results (see the discussion in [9]).

To reduce the effect of the statistical fluctuations it has been proposed [3] to fit the density $\rho(x)$ with a polynomial in $x$, which can be chosen to be an even polynomial here, since $\rho(x)$ is even. We implemented this suggestion in our analysis of the results for $\left\langle M-M^{*}\right\rangle$ and indeed we find a drastic improvement of the agreement with the reference data from the dual simulation (see [9] for the details). We stress at this point that the polynomial fit is not an essential ingredient of DoS FFA (or any other DoS variant), but simply an efficient method for reducing the effect of statistical errors. Alternatively one could also considerably increase the statistics of the Monte Carlo simulation, however, at a much higher computational cost.

\section{U(1) lattice gauge theory}

In this section we very briefly discuss the case of U(1) lattice gauge theory in 4 dimensions, which obviously is a theory without a complex action problem. Thus here the DoS FFA method is applied in a more conventional setting where the density is integrated with a non-oscillating Boltz- 

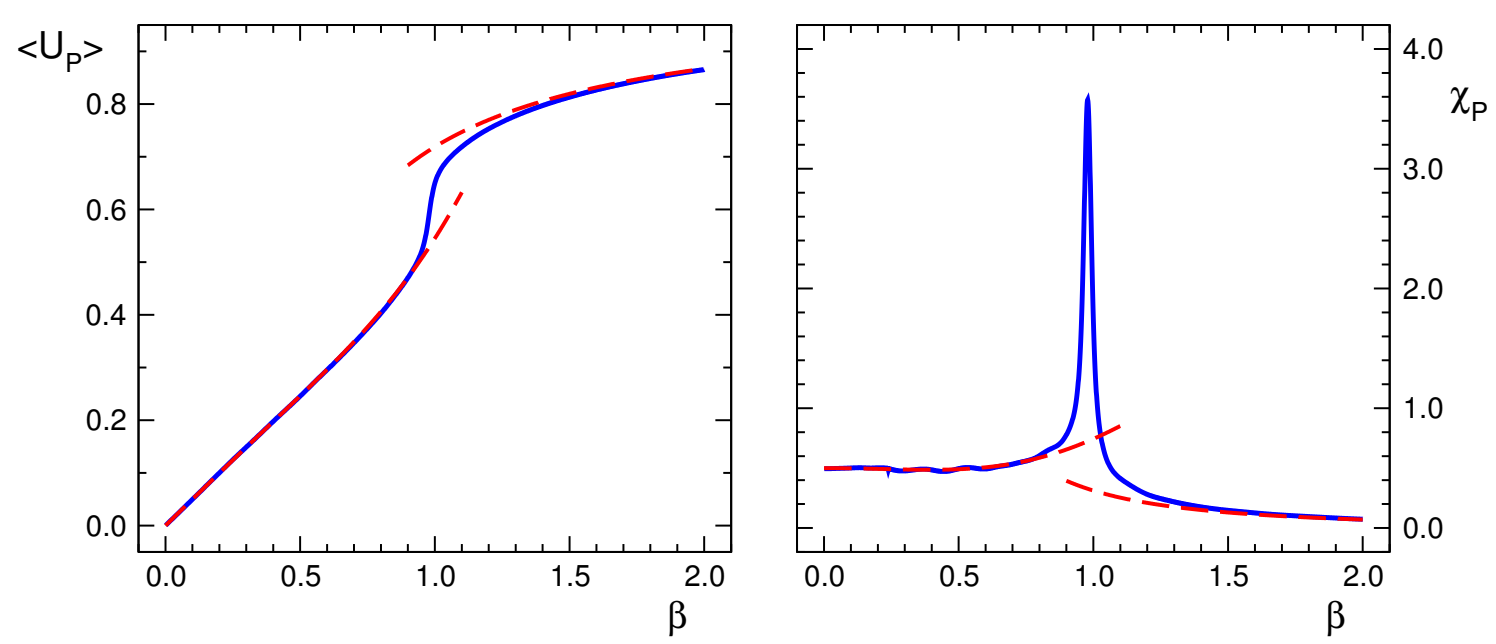

Figure 5: Results for the the plaquette expectation value and the plaquette susceptibility as a function of $\beta$. We show the results from DoS FFA (full curves) and from strong- and weak coupling expansions (dashed) taken from [11].

mann factor. Nevertheless, also here the numerics is challenging since the Boltzmann factor and the density vary over many orders of magnitude. The degrees of freedom are the gauge variables $U_{\mu}(x) \in \mathrm{U}(1)$ on the links of a 4-d lattice with periodic boundary conditions. For the action we use the usual Wilson plaquette action

$$
S_{G}[U]=-\beta \sum_{x} \sum_{\mu<v} \operatorname{Re} U_{\mu}(x) U_{v}(x+\hat{\mu}) U_{\mu}(x+\hat{v})^{\star} U_{v}(x)^{\star} .
$$

The partition sum is given by $Z=\int D[U] e^{-S[U]}$, where $D[U]$ is the product over the $\mathrm{U}(1)$ Haar measures over all links of the lattice.

For the implementation of DoS FFA according to the general scheme discussed in Section 2, we set $S_{\rho}=0$, i.e., we use a density $\rho(x)$ without any weight factor and choose $\xi X[U]=S_{G}[U]$ with $\xi=\beta$. The parameterization of $\rho(x)$ is chosen as given in (2.9) and (2.10). The determination of the parameters $k_{n}$ of $\rho(x)$ was implemented as described in Section 2. For a similar implementation of a DoS analysis of 4-dimensional U(1) lattice gauge theory with the DoS LLR approach see the last reference in [3].

The observables we study here are the plaquette expectation value $\left\langle U_{P}\right\rangle$ and the corresponding susceptibility $\chi_{P}$ defined as

$$
\left\langle U_{P}\right\rangle=\frac{1}{6 V} \frac{\partial \ln Z}{\partial \beta} \quad, \quad \chi_{P}=\frac{\partial\left\langle U_{P}\right\rangle}{\partial \beta},
$$

where $V$ denotes the number of lattice sites.

In Fig. 5 we show our results for $\left\langle U_{P}\right\rangle$ and $\chi_{P}$ as a function of $\beta$ for a small lattice $\left(V=4^{4}\right)$ and compare the DoS FFA results (full curves) to the weak and strong coupling results from [11] (dashed curves). Our results show the well known (first order) transition near $\beta=1.0$, and in the small- and large $\beta$ limits we find good agreement with the strong- and weak coupling results. 


\section{U(1) lattice gauge theory in 2 dimensions with a topological term}

Let us now finally come to an application of the DoS FFA to a class of systems where we expect a large potential for the DoS approach, i.e., models with a topological charge term as already outlined in the example given in Eq. (2.4). In particular the fact that the vacuum angle $\theta$ is restricted to the range $\theta \in[0, \pi]$ makes the DoS approach interesting, since in the integral over the density the oscillation frequency in the integrand $\rho(x) \cosh (\xi x)$ is linear in $\theta$ since $\xi=i \theta$, while for the chemical potential applications it is exponential in $\mu$ since $\xi=i \sinh \mu$ (compare Eq. (2.3)).

In this exploratory study we consider a system that is simpler than the Yang-Mills case sketched in (2.4), namely the case of $U(1)$ lattice gauge theory in 2 dimensions with a topological term. On the lattice the gauge action $S_{G}[U]$ and the topological charge $Q[U]$ in the field theoretical definition can be written as (see [10] for our conventions for $Q[U]$ ):

$$
S_{G}[U]=-\frac{\beta}{2} \sum_{x}\left[U_{x, p}+U_{x, p}^{*}\right] \quad, \quad Q[U]=\frac{1}{i 4 \pi} \sum_{x}\left[U_{x, p}-U_{x, p}^{*}\right],
$$

where the sums run over all sites $x$ of a 2-dimensional $N \times N=V$ lattice with periodic boundary conditions. $U_{x, p}=U_{x, 1} U_{x+\hat{1}, 2} U_{x+\hat{2}, 1}^{*} U_{x, 2}^{*}$ are the plaquettes made from the link variables $U_{x, v} \in$ $\mathrm{U}(1)$. The continuum limit is obtained by $\beta \rightarrow \infty, V \rightarrow \infty$ at fixed ratio $\beta / V=$ const, and we stress that only in this limit the partition $\operatorname{sum} Z(\theta)=\int D[U] e^{-S_{G}[U]-i \theta Q[U]}$ becomes $2 \pi$-periodic [10]. We remark that $\mathrm{U}(1)$ lattice gauge theory in 2 dimensions with a geometric definition of the topological charge $Q[U]$ was studied in [12], using a Monte Carlo approach that includes a trial distribution for the topological charges and Metropolis updates connecting neighboring charge sectors.

The transcription of the system to the general DoS FFA scheme as outlined in Section 2 is obvious via the identifications $S_{\rho}[U]=S_{G}[U], X[U]=Q[U]$ and $\xi=i \theta$. The parametrization we use is given by (2.9) and (2.10) and implementing the FFA scheme for determining the $k_{n}$ as outlined in Section 2.3 is straightforward.

An important aspect for the choice of the model (6.1) as a test case for systems with a vacuum angle is the fact that, based on a dual variable approach, one can write the partition $\operatorname{sum} Z$ as [10]

$$
Z(\theta)=\sum_{q=-\infty}^{+\infty}\left[I_{|q|}(2 \sqrt{\eta \bar{\eta}})\left(\sqrt{\frac{\eta}{\bar{\eta}}}\right)^{q}\right]^{V}
$$

where the $I_{n}$ are the modified Bessel functions and we define $\eta \equiv \frac{\beta}{2}-\frac{\theta}{4 \pi}, \bar{\eta} \equiv \frac{\beta}{2}+\frac{\theta}{4 \pi}$. Explicit expressions for the topological charge density and the topological susceptibility are then obtained as derivatives

$$
\langle q\rangle \equiv \frac{-1}{V} \frac{\partial}{\partial \theta} \ln Z \quad, \quad \chi_{t} \equiv \frac{-1}{V} \frac{\partial^{2}}{\partial \theta^{2}} \ln Z,
$$

while for the representation in terms of the density of states we again use (2.8). However, the explicit expression (6.2) even allows for an expression for the density $\rho(x)$ as a Fourier transform,

$$
\rho(x)=\int d \theta e^{i \theta x} Z(\theta)=\sum_{q=-\infty}^{+\infty} \int d \theta e^{i \theta x}\left[I_{|q|}(2 \sqrt{\eta \bar{\eta}})\left(\sqrt{\frac{\eta}{\bar{\eta}}}\right)^{q}\right]^{V} .
$$

The expression (6.4) can be evaluated numerically with Mathematica and in this presentation we focus on the density $\rho(x)$ for assessing the quality of the results from DoS FFA. 

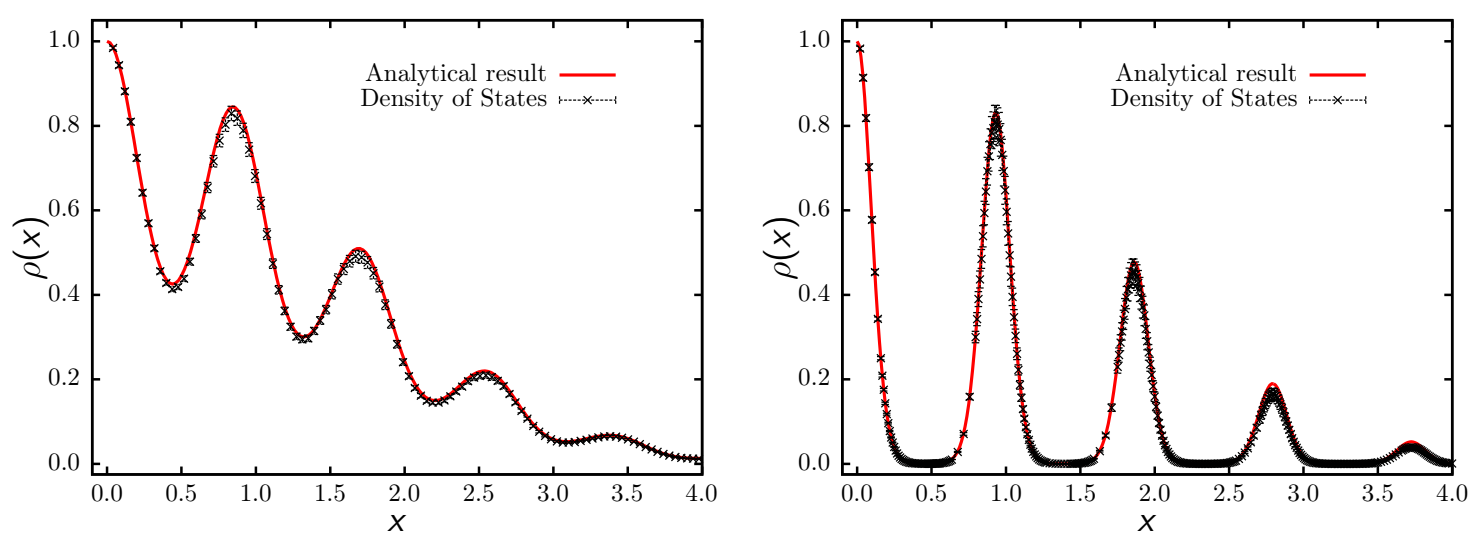

Figure 6: The density $\rho(x)$ as a function of $x$ for two different volumes and values of $\beta: 20 \times 20, \beta=4.0$ in the lhs. plot and $28 \times 28, \beta=7.84$ on the rhs. The crosses represent the results from DoS FFA and the full curve the exact result from Eq. (6.4).

In this proceedings contribution we present only preliminary data from an exploratory study of the DoS FFA for U(1) lattice gauge theory with topological term. More specifically we use two lattice volumes $20 \times 20$ and $28 \times 28$ at $\beta=4.0$ and $\beta=7.84$, such that $\beta / V=0.01=$ const. We currently work on results for bigger lattices and larger $\beta$, such that the system is closer to the continuum limit. In Fig. 6 we show the DoS FFA results (crosses) for the density $\rho(x)$ as a function of $x$. The continuous curves are the reference results from (6.4).

It is obvious that the DoS FFA data agree very well with the reference curves from (6.4). Only near the extrema of $\rho(x)$ we observe small deviations. These are due to the fact that there the slopes $k_{n}$ used in the parameterization (2.9)) are changing rapidly near the extrema. Thus the observed discrepancy is a hint that near the extrema smaller interval sizes $\Delta_{n}$ should be used - an improvement we are currently testing.

We have already remarked that the continuum limit is approached by sending $V$ and $\beta$ to infinity at a fixed ratio $\beta / V=$ const, and only in this limit the observables become $2 \pi$-periodic. As a consequence one expects that $\rho(x)$ becomes sharply peaked at integer values of $x$. The two datasets shown in Fig. 6 both correspond to $\beta / V=0.01$, with the rhs. data at $V=28 \times 28, \beta=7.84$ being closer to the continuum limit than the lhs. with $V=20 \times 20, \beta=4.00$. It is obvious that for the rhs. plot the density is indeed more peaked and in between the peaks $\rho(x)$ is approaching 0 . Still we find a good description of the density from (6.4) by the data from DoS FFA, despite the fact that here we can present only very preliminary data.

We stress at this point that regions with vanishing $\rho(x)$ do not pose a problem for the DoS FFA. The actual simulation is done with the ensemble (2.11) which is modified by the additional Boltzmann factor $e^{\lambda X}$ and the parameters of the density are determined from fitting the expectation values (2.14) (compare also Fig. 1). The fact that $\rho(x)$ may vanish for some $x$ has no influence on that procedure. We are currently improving the technology for the application of DoS FFA to theories with a topological term and in particular study the performance of the DoS FFA in the 2-dimensional U(1) theory with vacuum term closer to the continuum limit. 


\section{Concluding remarks}

In this proceedings contribution we give an overview over recent developments for the DoS FFA method and its application in various systems. The DoS FFA method is characterized by a parameterization of the density of states $\rho(x)$ on small intervals of its argument $x$. Restricted Monte Carlo simulations are performed on these intervals of size $\Delta_{n}$ and the system is probed with an additional Boltzmann factor $e^{\lambda x}$. Varying $\lambda$ explores all possible states in the given interval of $x$ and the parameters used in the parameterization of $\rho(x)$ in that interval can be determined by fitting the Monte Carlo data with a known function of $\lambda$. We stress that the method makes use of all Monte Carlo data that were created for the different values of $\lambda$, as they all contribute to the fit where the parameters of $\rho(x)$ are determined. Furthermore, one can also analyze the quality of that fit. If the quality is poor this is a signal that the chosen parameterization is too coarse and the $\Delta_{n}$ should be smaller. Thus the DoS FFA comes with a consistency check provided by the quality of the fit of the restricted Monte Carlo data with the known function of $\lambda$.

The method was tested in four different systems with different types of complex action problems and different technical challenges. The first system we explore is the SU(3) spin system with a chemical potential. The real part of the action is included in the weighted density of states which is determined in a straightforward way with the DoS FFA strategy. We show that the restricted expectation values are well described by the fit function - and the quality of the fit indicates that the density is properly described by the chosen parameterization and interval size. We present results for the particle number and its susceptibility and find good agreement with the reference data from a dual simulation for up to at least $\mu \beta \sim 2.5$ - quite remarkable for a first test with low statistics.

The next system we analyzed is the $Z_{3}$ system where the degrees of freedom are discrete spins. Thus the imaginary part of the action can have only discrete values and an exact parameterization can be chosen for $\rho(x)$ in the form of a piecewise constant ansatz. Here one uses overlapping restricted simulations on neighboring intervals and determines the constants again by fitting a known function of $\lambda$. For the free energy we here find perfect agreement with the dual reference simulation up to $\mu \beta \sim 4.0$, while for the imaginary part of the magnetization (related to the particle number) we find that for our $\tau=0.16$ ensemble a fit of the density with a polynomial was needed to achieve the same accuracy.

A small test simulation was implemented for pure $\mathrm{U}(1)$ gauge theory in 4 dimensions which has no complex problem such that a density without weights was used and the numerical challenge comes from accurately integrating the density over many orders of magnitude. Also in this case of a simple gauge theory we find good performance of the DoS FFA method.

Finally we applied the DoS FFA method to a model system where the complex action problem is due to a topological term: $\mathrm{U}(1)$ gauge theory in two dimensions with a topological term. We believe that this type of systems are interesting for DoS techniques, since the frequency of the oscillating factor grows linearly with the vacuum angle $\theta$, while for systems with a chemical potential $\mu$ the frequency grows exponentially in $\mu$. However, here other challenges have to be dealt with, in particular a density $\rho(x)$ which is non-monotonical and develops Dirac delta like behavior in the continuum limit. Thus one needs a very fine parameterization of $\rho(x)$ near the peaks of the density. In the exploratory feasibility study presented here we show that these challenges can successfully be dealt with and we find good agreement of the density from DoS FFA with the analytical result. 


\section{References}

[1] Z. Fodor, S. D. Katz and C. Schmidt, The density of states method at non-zero chemical potential, JHEP 0703 (2007) 121 [hep-lat/0701022].

[2] H. Saito, S. Ejiri, S. Aoki, K. Kanaya, Y. Nakagawa, H. Ohno, K. Okuno and T. Umeda, Histograms in heavy-quark QCD at finite temperature and density, Phys. Rev. D 89 (2014) 3, 034507 [arXiv:1309.2445 [hep-lat]]. S. Ejiri, Phase structure of hot dense QCD by a histogram method, Eur. Phys. J. A 49 (2013) 86 [arXiv:1306.0295 [hep-lat]]. S. Ejiri et al. [WHOT-QCD Collaboration], Numerical study of QCD phase diagram at high temperature and density by a histogram method, Central Eur. J. Phys. 10 (2012) 1322 [arXiv:1203.3793 [hep-lat]].

[3] K. Langfeld, B. Lucini, R. Pellegrini and A. Rago, An efficient algorithm for numerical computations of continuous densities of states, arXiv:1509.08391 [hep-lat]; K. Langfeld and B. Lucini, Density of states approach to dense quantum systems, Phys. Rev. D 90 (2014) 9, 094502 [arXiv:1404.7187 [hep-lat]]; K. Langfeld, B. Lucini and A. Rago, The density of states in gauge theories, Phys. Rev. Lett. 109 (2012) 111601 [arXiv:1204.3243 [hep-lat]].

[4] J. Greensite, J. C. Myers and K. Splittorff, The density in the density of states method, JHEP 1310 (2013) 192 [arXiv:1308.6712 [hep-lat]].

[5] C. Gattringer and P. Törek, Density of states method for the $\mathbb{Z}_{3}$ spin model, Phys. Lett. B 747 (2015) 545 [arXiv:1503.04947 [hep-lat]]; Y. Delgado Mercado, P. Törek and C. Gattringer, The $\mathbb{Z}_{3}$ model with the density of states method, PoS LATTICE 2014 (2015) 203 [arXiv:1410.1645 [hep-lat]].

[6] F. Wang and D.P. Landau, Efficient, Multiple-Range Random Walk Algorithm to Calculate the Density of States, Phys. Rev. Lett. 86 (2001) 2050.

[7] C. Gattringer, Flux representation of an effective Polyakov loop model for QCD thermodynamics, Nucl. Phys. B 850 (2011) 242 [arXiv:1104.2503 [hep-lat]].

[8] Y. D. Mercado and C. Gattringer, Monte Carlo simulation of the SU(3) spin model with chemical potential in a flux representation, Nucl. Phys. B 862 (2012) 737 [arXiv:1204.6074 [hep-lat]]; Y. Delgado and C. Gattringer, Flux simulation of the SU(3) spin model at finite chemical potential, Acta Phys. Polon. Supp. 5 (2012) 1033 [arXiv:1208.1169 [hep-lat]].

[9] Y. D. Mercado, H. G. Evertz and C. Gattringer, The QCD phase diagram according to the center group, Phys. Rev. Lett. 106 (2011) 222001 [arXiv:1102.3096 [hep-lat]]; Y. D. Mercado, H. G. Evertz and C. Gattringer, Worm algorithms for the 3-state Potts model with magnetic field and chemical potential, Comput. Phys. Commun. 183 (2012) 1920 [arXiv:1202.4293 [hep-lat]].

[10] C. Gattringer, T. Kloiber and M. Müller-Preussker, Dual simulation of the 2-dimensional lattice U(1) gauge-Higgs model with a topological term, arXiv:1508.00681 [hep-lat]; T. Kloiber and C. Gattringer, Scalar $Q E D_{2}$ with a topological term - a lattice study in a dual representation, PoS LATTICE2014 (2014) 345 [arXiv:1410.3216 [hep-lat]].

[11] B.E. Lautrup and M. Nauenberg, Phase Transition in Four-Dimensional Compact QED, Phys. Lett. B 95 (1980) 63.

[12] U. J. Wiese, Numerical Simulation of Lattice $\theta$ Vacua: The 2-d U(1) Gauge Theory as a Test Case, Nucl. Phys. B 318 (1989) 153. 